\title{
Perioperative Medication Management for Spinal Surgery
}

\author{
Nicholas S Venuti ${ }^{1}$, Sangili Chandran ${ }^{2}$, Connor Willis-Hong ${ }^{1}$ and \\ Vivek Mohan ${ }^{1 *}$ \\ ${ }^{1}$ Orthopaedic Spine Institute, Hoffman Estates, IL, USA \\ ${ }^{2}$ Advocate Medical Group, Family Medicine Associate Professor, Rosalind \\ Franklin University, Chicago, IL, USA \\ *Corresponding Author: Vivek Mohan, Orthopaedic Spine Institute, Hoffman \\ Estates, IL, USA.
}

Received: March 29, 2020

Published: April 10, 2020

(c) All rights are reserved by Vivek Mohan., et al.

\begin{abstract}
Background: Perioperative medication management of spinal surgery patients is essential to minimize risk of complications and expedite patient recovery. Commonly prescribed medication regimens such as anticoagulants (e.g. aspirin, clopidogrel), nonsteroidal anti-inflammatory drugs (e.g. ibuprofen, naproxen) and immunosuppressants (e.g. methotrexate, cyclosporine) may predispose patients to excessive hemorrhaging, wound dehiscence, and surgical infections among other intra-operative and post-operative complications. Through an understanding of medication mechanisms, recommended use and disuse protocol, and how these medications pertain to individual circumstances, physicians are optimally informed to prepare patients for elective spinal surgery. Study Design: Review of literature.

Methods: Numerous searches were conducted utilizing PubMed. The searches were filtered to be written in English and within twenty years.

Results: After review of relevant literature, different precautions must be taken depending on the type of medication (anti-coagulant, anti-platelet, non-steroidal anti- inflammatory drugs, immunosuppressants) and the individual perioperative health conditions. Patients on blood thinning regimens may need to discontinue use of prescribed medication as early as 10 days to 12 hours preoperatively and may resume consumption as early as 12 hours post-operatively depending on the medication and perioperative health condition of the patient. Patients on nonsteroidal anti-inflammatory drugs (NSAIDs) may need to discontinue use as early as eight days to 12 hours pre-operatively and may not resume consumption of NSAIDs for up to three months post-operatively depending on medication and the patient's perioperative health condition. Patients on immunosuppressant regimens may need to discontinue use as early as two months to eight hours pre-operatively and may resume post-operative consumption as early as one week or when the operative wounds have completely healed depending on the medication and the patient's perioperative health condition.

Conclusion: Due to the invasive nature of spinal surgery, and the potential effect of various medications that can affect surgical outcomes, it is imperative that providers review patient medications for proper management during the perioperative period. In the future, additional research for new classes of drugs and medications where literature is currently scarce will help to reduce hospital admission lengths, complications post-operation and even death.
\end{abstract}

Keywords: Perioperative Pain Management; Anti-Coagulants; Anti-Platelets; Nonsteroidal Anti-Inflammatory Drugs; Immunosuppressants; Spine Surgery

\section{Introduction}

With a rising incidence of chronic back pain and associated postural maladies, more patients are undergoing elective spinal procedures. Patients with pre-existing or comorbid medical conditions may require medication regimens that uniquely predispose them to complications resulting in increased costs, prolonged recoveries, or even death. Therefore, it is imperative that medications are both monitored and managed appropriately. This review article serves to provide a review of perioperative medication management- with a primary focus on non-pain medications- to be considered as physicians prepare patients for elective spinal surgery. Due to the frequency by which these medications are prescribed and the severity of the complications they may pose, the three main categories covered are Anticoagulants, Nonsteroidal Anti-Inflammatory Drugs (NSAIDs), and Immunosuppressants. This article will cover risks and general guidelines for each group as well as specific protocols for the most commonly used medications within each category.

\section{Anticoagulants and anti-platelets (Blood thinners)}

Patients with a history of heart disease or hypercoagulable states often follow a strict, daily regimen of blood thinners to prevent thrombosis. When in need of surgery, however, these patients face the dilemma of stopping or continuing their medication, both 
of which has their own associated risk. If ceasing treatment prior to surgery is decided, there remains an increased risk of thromboembolism exacerbated by the subsequent, rebound-hypercoagulable state and prolonged immobility during and following surgery [1]. Conversely, if the medications are administered up to the time of surgery, the patient is at an increased risk of hemorrhage intraoperatively. There is a delicate balance that must be maintained for each drug. The following outlines the appropriate measures to be taken with common blood thinner medications in order to avert these potential complications.

\section{Aspirin: Anti-platelet drug}

Aspirin, or Acetylsalicylic Acid (ASA), inhibits platelet function through irreversible COX-enzyme inhibition [2]. As a potent anti-thrombotic, aspirin is the most common drug used to prevent thromboembolic emergencies, as well as reduce the risk of myocardial infarctions and strokes [3,4]. Pre-operative discontinuation should be considered for patients at low risk for thrombosis (i.e. 6 months after MI without complications, or 12 months after stroke without complications) should discontinue aspirin use 7 - 10 days pre-operatively $[5,6]$. Patients at moderate-high risk for thrombosis (i.e. drug-eluting stent within 1 year, 2 weeks after stroke, or 6 weeks after MI) may either reduce their dosage to $81 \mathrm{mg} /$ day or continue aspirin use until surgery [5].

Post-operatively, Aspirin resumption within 24 hours of surgery has been shown to increase patient risk of major bleeding emergencies (e.g. hematomas) by $1.2 \%$. This value decreases to $0.7 \%$ when aspirin is resumed 5 days post-operatively, and to $0 \%$ about 10 days after surgery. Once hemostasis is attained postoperatively, the dosage should be returned to pre-operation dose [6-8]. Thus, aspirin resumption is dependent on patient circumstances, anticipated blood loss for surgical approach, and surgeon discretion [9].

Additionally, low-dose "baby" aspirin regimens (81 mg) are a common alternative to regular aspirin regimens $(325 \mathrm{mg}$ ) in an effort to avoid the gastrointestinal complications associated with regular aspirin use. The current literature regarding low-dose aspirin perioperative management is consistent with that of regulardose aspirin (see above). However, a few studies indicate risk of increased blood loss in patients taking low-dose aspirin, regardless of when the regimen is discontinued pre-operatively $[10,11]$. Therefore, low-dose aspirin management is dependent on patient circumstances and the surgeon's discretion.

\section{Apixaban (Eliquis): Anti-coagulant drug}

Apixaban is a direct clotting factor Xa inhibitor, it inhibits conversion of prothrombin to thrombin to prevent coagulation [4]. The recommended pre-operative discontinuation is dependent upon renal function. If the pre-operative serum creatinine level ( $\mathrm{SCr}$ ) is less than $1.5 \mathrm{mg} / \mathrm{dL}$, discontinuing 48 hours prior is sufficient. However, if $\mathrm{SCr}$ is greater than or equal to $1.5 \mathrm{mg} / \mathrm{dL}$, discon- tinuation should be 72 hours pre-operatively. Resuming Apixaban post-operatively may resume within 72 hours, but caution must be taken due to Apixaban's rapid onset and offset $[8,12]$.

\section{Clopidogrel (Plavix): Anti-platelet drug}

Clopidogrel inhibits platelet aggregation through irreversible inhibition of the ADP-mediated P2Y12 receptor [4]. According to the CHEST guidelines, pre-operative discontinuation of Clopidogrel should be 7 - 10 days pre-operatively to allow for complete replenishment of patient platelet stores. It is recommended resuming Clopidogrel within 24 hours post-operatively or as soon as possible [6].

\section{Enoxaparin (Lovenox): Anti-coagulant drug}

Enoxaparin, a Low Molecular Weight Heparin (LMWH), accelerates the activity of antithrombin III, thus inhibiting clotting factors Xa and IIa [4]. Due to its short half-life (4.5 hours), Enoxaparin is typically used to bridge Warfarin. The prophylactic dosing (e.g. 40 mg once daily) should be discontinued 12 hours pre-operatively, and the therapeutic dosing (e.g. $1 \mathrm{mg} / \mathrm{kg}$ twice daily) should be discontinued 24 hours pre-operatively. Both may be resumed within 72 hours post-operatively, if approved by surgeon $[8,12]$.

\section{Warfarin (Coumadin): Anti-coagulant drug}

Warfarin prevents coagulation by preventing synthesis of clotting factors II (prothrombin), VII, IX, and X [4]. Warfarin is typically discontinued 5 days pre-operatively or longer to allow for patient INR normalization (INR $\leq 1.4$ the day before surgery). If the INR remains $>1.5$, a low-dose oral Vit. K supplement may be administered to hasten INR normalization [7,12]. Warfarin should be resumed 12 - 24 hours after surgery at the pre-operative dosage $[5,12]$. Patient INR values require 5 - 10 days after restarting warfarin therapy to reach appropriate levels; thus these patients must be kept under close observation or undergo bridging therapy as they may be at risk for thromboembolic emergencies.

Bridging anti-coagulation minimizes the time to reach therapeutic range in order to reduce risk of thromboembolism. However, bridging increases the patient's risk of post-operative bleeding, and the physician must decide whether bridging is appropriate based on individual circumstances. If necessary, a course of subcutaneous LMWH is started three days pre-operatively [12]. This method allows for a precise window of normal hemostasis during which surgery may be conducted; once the surgical hemorrhagic risk has passed, anticoagulation therapy may be resumed [7]

\section{Non-steroidal anti-inflammatory drugs (NSAIDS)}

NSAIDs, a class of COX-enzyme-inhibiting drugs, are commonly used to treat chronic conditions (e.g. osteoarthritis, rheumatoid arthritis) or post-operative pain management for their analgesic, anti-inflammatory, and anti-pyretic effects [13]. NSAIDs were traditionally produced as non-specific COX inhibitors that produced many unwanted side effects, most frequently gastrointestinal 


\begin{tabular}{|c|c|c|c|}
\hline Medication & Pre-Operative Discontinuation & Post-Operative Resumption & Bridging** \\
\hline Aspirin & $\begin{array}{l}\text { - Low Risk Patients: } 7 \text { - } 10 \text { days pre-operatively. } \\
\text { - Moderate-High Risk Patients: May continue } \\
\text { aspirin use until surgery. }\end{array}$ & $\begin{array}{l}\text { Within } 24 \text { hours if approved } \\
\text { by surgeon. }\end{array}$ & \\
\hline $\begin{array}{l}\text { Apixaban } \\
\text { (Eliquis) }\end{array}$ & $\begin{array}{l}\text { - } \mathrm{SCr}<1.5 \mathrm{mg} / \mathrm{dL}: 48 \text { hours pre-operatively. } \\
\text { - } \mathrm{SCr} \geq 1.5 \mathrm{mg} / \mathrm{dL}: 72 \text { hours pre-operatively. }\end{array}$ & $\begin{array}{l}\text { Within } 72 \text { hours if approved } \\
\text { by surgeon. }\end{array}$ & \\
\hline $\begin{array}{l}\text { Clopidogrel } \\
\text { (Plavix) }\end{array}$ & 7 - 10 days pre-operatively. & $\begin{array}{l}\text { Within } 24 \text { hours or as soon as } \\
\text { possible. }\end{array}$ & \\
\hline $\begin{array}{l}\text { Enoxaparin } \\
\text { (Lovenox) }\end{array}$ & $\begin{array}{l}\text { - Prophylactic Dosing: } 12 \text { hours pre-operatively. } \\
\text { - Therapeutic Dosing: } 24 \text { hours pre-operatively. }\end{array}$ & $\begin{array}{l}\text { Within } 72 \text { hours if approved } \\
\text { by surgeon. }\end{array}$ & \\
\hline $\begin{array}{l}\text { Warfarin } \\
\text { (Coumadin) }\end{array}$ & $\begin{array}{l}5 \text { days pre-operatively provided the patient's INR } \leq \\
1.4 \text { the day before surgery. }\end{array}$ & $\begin{array}{l}12 \text { - } 24 \text { hours after surgery at } \\
\text { pre-operative dosage. }\end{array}$ & $\begin{array}{c}\text { If necessary, subcutaneous LMW } \\
\text { Heparin is started } 3 \text { days pre- } \\
\text { operatively. }\end{array}$ \\
\hline
\end{tabular}

Table 1: Blood thinner use overview.

**: Bridging is typically reserved for patients taking warfarin and at high risk of thromboembolism

(i.e. embolic event within the previous 3 months, mechanical mitral/aortic valve, atrial fibrillation and high risk of stroke, etc.) [12].

\section{Ibuprofen (Advil, Motrin, Midol, Nuprin, Pamprin)}

Ibuprofen, a non-specific NSAID, functions through inhibition of both COX-1 and COX-2 enzymes [19]. Ibuprofen is further categorized as a "short-acting" NSAID, characterized by a half-life $\leq$ 6 hours [13]. A study observing the effects of common NSAIDs on human subjects determined the duration of each medication's anti-platelet activity (non-specific NSAIDs block ADP- or epinephrine-induced platelet aggregation). Once the patient's platelet aggregation abilities returned, it was assumed that the medication had been flushed from the patient's system. Ibuprofen's effects dissipated within 24 hours of ingestion, and thus can be stopped 24 hours pre-operatively [20].

An animal study determined that, if NSAIDs are resumed $\geq 4$ weeks post-operatively, NSAID inhibitory effects on bone healing were negligible [21]. The relatively few human studies performed point to significantly lower fusion success rates in patients who resume NSAIDs $<3$ months post-operatively. Thus, all NSAIDs are typically resumed $\geq 3$ months post-operatively or when approved by the surgeon [22].

\section{Diflunisal}

A non-specific NSAID, Diflunisal inhibits both COX-1 and COX2 enzymes. Diflunisal is a "long-acting" NSAID, with a half-life $\geq 6$ hours [23]. An early study observed that Diflunisal's effects dissipated within 24 hours of ingestion [20]. However, a subsequent, more detailed study determined that Diflunisal's effects were dose-dependent. Daily doses of $1000 \mathrm{mg}$ of Diflunisal (as opposed to $250 \mathrm{mg}$ or $500 \mathrm{mg}$ doses) impaired platelet aggregation similar to that of aspirin, with effects subsiding after 8 days. Thus, Diflunisal may be discontinued 24 hours pre-operatively for 250 - 500 $\mathrm{mg} /$ day doses and $\geq 8$ days pre-operatively for $1000 \mathrm{mg} /$ day doses if approved by the surgeon [2,24]. Post-operative resumption is suggested at $\geq 3$ months post-operatively or when approved by the surgeon [22].

\section{Diclofenac (Arthrotec, Cataflam, Voltaren)}

A non-specific NSAID, Diclofenac inhibits both COX-1 and COX-2 enzymes [19]. Diclofenac is a "short-acting" NSAID, with a half-life $\leq 6$ hours [13]. Diclofenac's effects dissipated 2 days after ingestion, and thus can be stopped $\geq 2$ days pre-operatively [20]. They may be safely resumed $\geq 3$ months post-operatively or when approved by the surgeon [22].

\section{Indomethacin (Indocin)}

Indomethacin is a non-specific NSAID, that inhibits both COX-1 and COX-2 enzymes [19]. Indomethacin is a "short-acting" NSAID, with a half-life $\leq 6$ hours [13]. Early studies of Indomethacin use revealed that clearance correlated to the logarithm of diminishing Indomethacin plasma levels [25]. With subsequent experimentation, it was determined that Indomethacin's effects were adequately dissipated 2 days after ingestion [20]. Thus, Indomethacin can be stopped 2 days pre-operatively. Recommended post-operative resumption should be $\geq 3$ months post-operatively or when approved by the surgeon [22].

\section{Naproxen (Aleve, Anaprox, Naprosyn)}

Naproxen is a non-specific NSAID, which inhibits both COX-1 and COX-2 enzymes [19]. Naproxen is a "long-acting" NSAID, with a half-life $\geq 6$ hours [13]. It's effects are dissipated 2 days after ingestion, and thus can be stopped $\geq 2$ days pre-operatively [20]. Naproxen may be resumed $\geq 3$ months post-operatively or when approved by the surgeon [22].

\section{Piroxicam (Feldene)}

A non-specific NSAID, Piroxicam inhibits both COX-1 and COX2 enzymes. Piroxicam is a "long-acting" NSAID, with a half-life $\geq 6$ hours [13]. Piroxicam's effects dissipated 3 days after ingestion, and thus can be stopped $\geq 3$ days pre-operatively [20]. It also may be resumed $\geq 3$ months post-operatively or when approved by the surgeon [22]. 


\begin{tabular}{|c|c|c|}
\hline Medication & Pre-Operative Discontinuation & Post-Operative Resumption \\
\hline $\begin{array}{l}\text { Ibuprofen (Advil, Motrin, Midol, Nuprin, } \\
\text { Pamprin) }\end{array}$ & $\geq 24$ hours pre-operatively. & \multirow[t]{6}{*}{$\begin{array}{l}\geq 3 \text { months post-operatively } \\
\text { or when approved by surgeon. }\end{array}$} \\
\hline Diflunisal & $\begin{array}{c}-250 \mathrm{mg} / \text { day or } 500 \mathrm{mg} / \text { day Doses: } \\
\geq 24 \text { hours pre-operatively. } \\
-1000 \mathrm{mg} / \text { day Doses: } \\
\quad 8 \text { days pre-operatively if approved } \\
\text { by surgeon. }\end{array}$ & \\
\hline $\begin{array}{l}\text { Diclofenac (Arthrotec, Cataflam, } \\
\text { Voltaren) }\end{array}$ & $\geq 2$ days pre-operatively. & \\
\hline Indomethacin (Indocin) & $\geq 2$ days pre-operatively. & \\
\hline Naproxen (Aleve, Anaprox, Naprosyn) & $\geq 2$ days pre-operatively. & \\
\hline Piroxicam (Feldene) & $\geq 3$ days pre-operatively. & \\
\hline
\end{tabular}

Table 2: Non-specific NSAID use overview.

\section{COX-2 antagonist use and bone fusion rates}

The relatively recent advent of COX-2 specific antagonists has not allowed for sufficient investigation to definitively determine their effects on post-operative bone fusion and healing rates. While several studies point to COX-2 antagonists impeding bone healing [15], others indicate that COX-2 specific NSAIDs have no lasting negative effects [26,27].

Studies concerning COX-2 antagonist use and bone fusion rates are even scarcer. Recent studies, however, provide promising evidence supporting the relative safety of post-operative COX-2 antagonist use (specifically Celecoxib) for acute pain management without compromising bone fusion rates. The first animal study to compare post-operative non-specific NSAID use to COX-2 antagonist use on spinal fusion rates found that post-operative indomethacin treatment significantly reduced fusion success rates from $64 \%$ in the control group to $18 \%$ in the indomethacin group; whereas the Celecoxib group showed a $45 \%$ fusion success rate that was not statistically different from the control group $[17,28]$.

The invasive nature of spinal surgery often leaves the patient in significant post-operative pain, experiencing difficulty with mobilization, and nauseated/vomiting- conditions that may prove overwhelming and therefore compromise rehabilitation [29,30]. Such concerns have prompted research into alternative methods to improve post-operative pain control, while decreasing narcotic usage. A recent meta-analysis comparing studies involving different NSAID-mediated post-operative regimens found that short time ( $<14$ days) exposure of high-dose ketorolac increased the risk of nonunion, whereas short time $(<14$ days) exposure of normaldose NSAIDs produced results similar to pain regimens lacking NSAIDs [31]. Such studies indicate NSAID effects on spinal fusion rates may be dose-dependent.

In light of such results, and the trend toward more outpatient spine procedures, there is great interest in the viability of multimodal pain regimens including NSAIDs that can successfully manage post-operative pain without increasing the likelihood of adverse effects and lead to earlier discharge [32]. One such study investigated the efficacy of a pre-emptive multimodal analgesic regimen in reducing post-operative pain and complications following primary lumbar fusion surgery. The study determined that patients receiving the experimental regimen (included Celecoxib) showed no difference in intra-operative blood loss, post-operative drain output, or non-union rates as well as lower post-operative pain and better functionality when compared to the control patient group receiving standard intravenous morphine [33]. Another study mirrored these findings and determined a comprehensive multimodal treatment regimen could significantly reduce morphine administration, improve mobilization, and shorten the length of stay under observation compared to the control population [30].

These are just a few examples of studies that, through obtaining a better understanding of the effects of COX- 2 antagonists, open a very promising branch of research that will have significant implications on post-operative pain management and surgical outcomes.

\section{Immunosuppressants}

Autoimmune diseases, characterized by the inappropriate destruction of a host's tissue by the host's immune system, are common chronic conditions. The most commonly seen autoimmune diseases in spinal surgery patients (perhaps even serving as the etiology of their spinal condition) include: Rheumatoid Arthritis, Ulcerative Colitis, Reiter's Syndrome, Ankylosing Spondylitis, etc.

Patients with such conditions often follow a strict regimen of immunosuppressants that may prove problematic when considering spinal surgery. The invasive nature of spinal surgery often dictates the need for immunosuppressants to prevent further exacerbation of the patient's inflammatory process. However, continued immunosuppressant use puts patients at an increased risk of autoimmune disease flares, wound infections (possibly leading to sepsis and death), and impaired wound healing [34].

Due to the relative scarcity of literature regarding perioperative management of immunosuppressant medications, it is difficult to provide definitive recommendations for use and disuse protocols. This article will serve to condense the most recent, agreed-upon literature regarding perioperative immunosuppressant management, and make recommendations accordingly. However, due to 
the systemic nature of autoimmune diseases, it is imperative that medication regimens are adjusted based on individual circumstances as well as the discretion of the patient's primary care physician, rheumatologist, and surgeon [34-36].

\section{Disease modifying anti-rheumatic drugs (DMARDs)}

Methotrexate (Trexall)

Methotrexate, an antifolate, works through inhibition of Dihydrofolate Reductase (thus impairing purine/pyrimidine metabolism), T-cell activation, and B-cell proliferation [37]. The majority of recent studies observing the perioperative effects of methotrexate management indicate no significant increases in post-operative infection rates or incidences of prolonged wound healing with methotrexate use $[38,39]$. However, if there are concerns regarding renal insufficiency (leading to buildup of methotrexate metabolites) or bone marrow suppression, methotrexate is typically discontinued 1 week pre-operatively [40]. If discontinued, methotrexate is resumed 1 week post-operatively or when the surgical wounds have successfully healed (when approved by the surgeon) [40].

\section{Cyclosporine (Neoral, Sandimmune)}

Cyclosporine, a T-lymphocyte specific immunosuppressant, blocks a calcium-dependent signal transduction pathway, thus preventing IL-2 transcription and interrupting T-cell activation [41]. As a potent T-cell inhibitor, cyclosporine perioperative use can present complications including opportunistic infections, seizures, and wound dehiscence. However, the available literature regarding cyclosporine perioperative management is quite scarce and contradictory. While one study implicates cyclosporine use in causing a significantly increased risk of post-operative complications (albeit through a coincidental analysis) [38], other studies investigating cyclosporine use and Inflammatory Bowel Disease found no increased risk of operative complications with pre-operative cyclosporine regimens [42]. As such, it is recommended that patients adhering to pre-operative cyclosporine regimens are carefully observed for deterioration in renal function or opportunistic infections. At the discretion of the surgeon and based upon individual patient conditions, cyclosporine may be discontinued relative to dosage amount and cyclosporine half-life (8.4 hours) [36]. If discontinued, cyclosporine is resumed when the surgical wounds have successfully healed (when approved by the surgeon) [40].

\section{Hydroxychloroquine (Plaquenil)}

Hydroxychloroquine increases the $\mathrm{pH}$ within intracellular vacuoles of antigen-presenting cells, thus altering intracellular processes and interfering with antigen processing in these cells [43]. It was subsequently postulated that hydroxychloroquine also inhibits the Toll-Like Receptor (TLR) 9 family of receptors- cellular receptors that mediate the inflammatory response [44]. Studies consistently note no increased risk of infectious complications and few potential side effects with Hydroxychloroquine continuation $[45,46]$. Thus, Hydroxychloroquine regimens are typically contin- ued during the perioperative period, if the patient can continue oral medications [47].

\section{Leflunomide (Arava)}

Leflunomide, a selective inhibitor of de novo pyrimidine synthesis, reversibly inhibits Dihydroorotate Dehydrogenase (DHODH). In doing so, Leflunomide prevents lymphocytes from accumulating sufficient pyrimidines for DNA synthesis, and thus proliferation [48]. The current literature presents conflicting data regarding Leflunomide perioperative management. While one study indicates no significant difference in infectious complications between patients who continued Leflunomide and those who did not [49], another study points to an increased risk of post-operative wound complication with Leflunomide continuation [50]. Thus, based on the discretion of the surgeon and primary care physician, Leflunomide is discontinued 2 months pre-operatively, if at all, due to its long half-life (1 - 4 weeks) [47]. If discontinued, Leflunomide is resumed 1 week post-operatively or when the surgical wounds have successfully healed (when approved by the surgeon) [47].

\section{Sulfasalazine (Azulfidiine)}

While the exact mechanism of action of Sulfasalazine in the treatment of autoimmune diseases has not yet been identified, it has been postulated that Sulfapyridine (an active agent in Sulfasalazine) reduces secretions of inflammatory cytokines such as interleukin (IL)-8 and monocyte chemoattractant protein (MCP)-1 [51]. Through retrospective review, Sulfasalazine is typically associated with a lower risk of perioperative infection [52]. Based on individual patient circumstances and the physician's discretion, Sulfasalazine is discontinued 1 week pre-operatively, if at all $[47,53]$ If discontinued, sulfasalazine is resumed when the surgical wounds have successfully healed (when approved by the surgeon) [47].

Tumor necrosis factor (TNF)- $\alpha$ antagonists: Etanercept, infliximab, adalimumab

TNF- $\alpha$, a systemic cytokine with a diversity of biological functions, plays a critical role in inducing lymphocyte proliferation and inflammation characteristic of rheumatoid arthritis and other autoimmune conditions that warrant the need for spinal surgery. The discovery of its role in autoimmune conditions prompted the advent of TNF- $\alpha$ antagonists including: Infliximab, Etanercept, and Adalimumab [54]. Infliximab (Remicade) is a monoclonal antibody specific for TNF- $\alpha$ that prevents the cytokine from triggering cellular TNF- $\alpha$ receptor complexes. Etanercept (Enbrel) is a chemical antagonist that binds to circulating TNF- $\alpha$ cytokines and deactivates them. Adalimumab (Humira) is a monoclonal antibody specific for TNF- $\alpha$ that prevents the cytokine from binding to cellular TNF- $\alpha$ receptors. Infliximab, Etanercept, and Adalimumab therapies are all most effective when administered in conjunction with Methotrexate [55].

Studies indicate a correlation between TNF- $\alpha$ inhibitor use and an increased risk of serious skin and soft tissue infections (SSTIs) in non-surgical patients (with no difference between the three major TNF- $\alpha$ inhibitor medications) [56]. This raises the concern that TNF- $\alpha$ antagonists may promote surgical site infections (a 
major cause of surgical morbidity). The current literature presents conflicting information regarding the complications TNF- $\alpha$ antagonist regimens may present during the perioperative period. While most studies indicate no statistically significant correlation between surgical site infection and TNF- $\alpha$ antagonist use/disuse $[57,58]$, a few associate TNF- $\alpha$ inhibitor therapy with serious postoperative infection (e.g. septic arthritis, osteomyelitis, deep wound infection) [59]. While clinical guidelines regarding TNF- $\alpha$ inhibitor perioperative management vary, it is generally recommended that the regimen is discontinued at least 2 weeks pre-operatively [47]. If discontinued, TNF- $\alpha$ antagonists are resumed 1 week postoperatively or when the surgical wounds have successfully healed (when approved by the surgeon) [59].

\section{Rituximab (Rituxan)}

Rituximab is a monoclonal antibody specific to the CD20 molecule on B-lymphocyte surfaces. Through mediation of antibody- dependent cellular cytotoxicity, complement-system activation, and B cell apoptosis, Rituximab effectively depletes mature B-cells and pre-B cells to depress immune function [60]. Through B-cell suppression, Rituximab therapy has been correlated with an increased incidence of perioperative complications including: deep/ superficial surgical infections, delayed wound healing, and opportunistic infections, with no significant link between the time of preoperative rituximab discontinuation and surgery [61]. For patients considering elective spinal surgery, it is advised that rituximab therapy is discontinued for $1-2$ treatment cycles pre-operatively to replenish B-cell stores. If dosed within 100 days of spinal surgery, Immunoglobulin (Ig) levels must be monitored, and supplemented with intravenous Ig if levels fall too low [62]. Rituximab may be resumed when the surgical wounds have successfully healed (when approved by the surgeon) [62].

\begin{tabular}{|c|c|c|}
\hline Medication & Pre-Operative Discontinuation & Post-0perative Resumption \\
\hline Methotrexate (Trexall) & $\begin{array}{c}1 \text { week pre-operatively (with concerns } \\
\text { of renal insufficiency or } \\
\text { myelosuppression). }\end{array}$ & $\begin{array}{c}1 \text { week post-operatively or when the surgical wounds have } \\
\text { successfully healed. }\end{array}$ \\
\hline $\begin{array}{l}\text { Cyclosporine (Neoral, Sand- } \\
\text { immune) }\end{array}$ & $\begin{array}{l}\text { Relative to dosage amount and c } \\
\text { yclosporine half-life ( } 8.4 \text { hours). }\end{array}$ & $\begin{array}{l}\text { If discontinued, cyclosporine is resumed when the surgical } \\
\text { wounds have successfully healed. }\end{array}$ \\
\hline $\begin{array}{l}\text { Hydroxychloroquine } \\
\text { (Plaquenil) }\end{array}$ & \multicolumn{2}{|c|}{ May be continued perioperatively. } \\
\hline Leflunomide (Arava) & 2 months pre-operatively, if at all. & $\begin{array}{l}1 \text { week post-operatively or when the surgical wounds have } \\
\text { successfully healed. }\end{array}$ \\
\hline Sulfasalazine (Azulfidiine) & 1 week pre-operatively, if at all. & $\begin{array}{l}\text { If discontinued, sulfasalazine is resumed when the surgical } \\
\text { wounds have successfully healed (at surgeon's discretion). }\end{array}$ \\
\hline $\begin{array}{l}\text { Tumor Necrosis Factor } \\
\text { (TNF)- } \alpha \text { Antagonists: Etan- } \\
\text { ercept, Infliximab, Adalim- } \\
\text { umab }\end{array}$ & $\geq 2$ weeks pre-operatively. & $\begin{array}{l}1 \text { week post-operatively or when the surgical wounds have } \\
\text { successfully healed (at surgeon's discretion). }\end{array}$ \\
\hline Rituximab (Rituxan) & $\begin{array}{l}\text { - } 1 \text { - } 2 \text { treatment cycles pre-operatively. } \\
\text { - If patient is dosed within } 100 \text { days } \\
\text { of spinal surgery, Ig levels must be } \\
\text { monitored and supplemented if levels } \\
\text { fall too low }\end{array}$ & $\begin{array}{l}\begin{array}{l}\text { When the surgical wounds have successfully healed (at sur } \\
\text { geon's discretion). }\end{array}\end{array}$ \\
\hline
\end{tabular}

Table 3: Immunosuppressant use overview.

\section{Conclusion}

Due to the invasive nature of spinal surgery, and the various medications that potentially effect spinal surgical outcomes, it is imperative that providers review patient medications for proper management during the perioperative period. In the future, additional research for new classes of drugs and medications where literature is currently scarce will help to reduce hospital admission lengths, complications post-operation, and even death.

\section{Disclosure}

The authors report no conflict of interest concerning the materials or methods used in this study or the findings specified in this paper.

\section{Bibliography}

1. Jaffer AK., et al. "When patients on warfarin need surgery". Cleveland Clinic Journal of Medicine 70.11 (2003): 973-984.

2. Schafer AI. "Effects of nonsteroidal anti-inflammatory drugs on platelet function and systemic hemostasis". The Journal of Clinical Pharmacology 35.3 (1995): 209-219.

3. Guirguis-Blake JM., et al. "Aspirin for the Primary Prevention of Cardiovascular Events: A Systematic Evidence Review for the US Preventive Services Task Force Aspirin for the Primary Prevention of Cardiovascular Events". Annals of Internal Medicine 164.12 (2016): 804-813. 
4. Hall R and Mazer CD. "Antiplatelet drugs: a review of their pharmacology and management in the perioperative period". Anesthesia and Analgesia 112.2 (2011): 292-318.

5. Douketis JD., et al. "Perioperative management of antithrombotic therapy: Antithrombotic Therapy and Prevention of Thrombosis, $9^{\text {th }}$ edition: American College of Chest Physicians Evidence-Based Clinical Practice Guidelines". Chest 141.2 (2012): e326S-e350S.

6. Mohr TS and Brouse SD. "Perioperative management of antiplatelet agents”. Orthopedics 35.8 (2012): 687-691.

7. Hill SE and D’Alonzo RC. "Perioperative Management of Bleeding and Transfusion, in Perioperative Medicine". Saunders Elsevier: Philadelphia PA (2008): 405-430.

8. Rose A. "Periprocedural and Regional Anesthesia Management with Antithrombotic Therapy- Adult - Inpatient and Ambulatory- Clinical Practice Guideline". Madison, WI: University of Wisconsin Health (2015).

9. Devereaux PJ., et al. "Aspirin in patients undergoing noncardiac surgery". The New England Journal of Medicine 370.16 (2014): 1494-1503.

10. Cuellar JM., et al. "Does Aspirin Administration Increase Perioperative Morbidity in Patients With Cardiac Stents Undergoing Spinal Surgery?" The Spine Journal 11.4 (2015): 629-635.

11. Kang SB., et al. "Does low-dose aspirin increase blood loss after spinal fusion surgery?" The Spine Journal 11.4 (2011): 303-307.

12. Lip GY and Douketis JD. "Perioperative Management of Patients Receiving Anticoagulants". UpToDate. Waltham, MA: UpToDate (2017).

13. Solomon DH. "NSAIDs: Pharmacology and Mechanism of Action". UpToDate. Waltham, MA: UpToDate (2017).

14. Langford RM and Mehta V. "Selective cyclooxygenase inhibition: its role in pain and anaesthesia". Biomed Pharmacotherapy 60.7 (2006): 323-328.

15. Cottrell J and O'Connor JP. "Effect of Non-Steroidal Anti-Inflammatory Drugs on Bone Healing". Pharmaceuticals (Basel) 3.5 (2010): 1668-1693.

16. Davidson BL., et al. "Bleeding risk of patients with acute venous thromboembolism taking nonsteroidal anti-inflammatory drugs or aspirin". JAMA Internal Medicine 174.6 (2014): 947-953.

17. Thaller J., et al. "The effect of nonsteroidal anti-inflammatory agents on spinal fusion". Orthopedics 28.3 (2005): 299-303.

18. Glassman SD., et al. "The effect of postoperative nonsteroidal anti-inflammatory drug administration on spinal fusion". Spine 23.7 (1998): 834-838.
19. McGettigan P and Henry D. "Current problems with nonspecific COX inhibitors". Current Pharmaceutical Design 6.17 (2000): 1693-1724

20. Cronberg S., et al. "Effect on platelet aggregation of oral administration of 10 non-steroidal analgesics to humans". Scandinavian Journal of Haematology 33.2 (1984): 155-159.

21. Riew KD., et al. "Time-dependent inhibitory effects of indomethacin on spinal fusion". Journal of Bone and Joint Surgery American 85-A.4 (2003): 632-634.

22. Deguchi M., et al. "Posterolateral fusion for isthmic spondylolisthesis in adults: analysis of fusion rate and clinical results". Journal of Spinal Disorders 11.6 (1998): 459-64.

23. Aschenbrenner DS and Venable SJ. "Drug Therapy in Nursing”. Philadelphia, PA: Wolters Kluwer Health/Lippincott Williams and Wilkins (2012)

24. Green D., et al. "Effects of diflunisal on platelet function and fecal blood loss”. Pharmacotherapy 3.2-2 (1983): 65S-69S.

25. Rane A., et al. "Relation between plasma concentration of indomethacin and its effect on prostaglandin synthesis and platelet aggregation in man". Clinical Pharmacology and Therapeutics 23.6 (1978): 658-668.

26. Karachalios T., et al. "The effects of the short-term administration of low therapeutic doses of anti-COX-2 agents on the healing of fractures. An experimental study in rabbits". The Journal of Bone and Joint Surgery British 89.9 (2007): 1253-1260.

27. Krischak GD., et al. "The non-steroidal anti-inflammatory drug diclofenac reduces appearance of osteoblasts in bone defect healing in rats". Archives of Orthopaedic and Trauma Surgery 127.6 (2007): 453-458.

28. Long J., et al. "The effect of cyclooxygenase-2 inhibitors on spinal fusion". Journal of Bone and Joint Surgery 84-A.10 (2002): 1763-1768.

29. Buvanendran A and Thillainathan V. "Preoperative and postoperative anesthetic and analgesic techniques for minimally invasive surgery of the spine". Spine 35.26 (2010): S274-S280.

30. Mathiesen 0., et al. "A comprehensive multimodal pain treatment reduces opioid consumption after multilevel spine surgery”. European Spine Journal 22.9 (2013): 2089-2096.

31. Li Q., et al. "High-dose ketorolac affects adult spinal fusion: a meta-analysis of the effect of perioperative nonsteroidal anti-inflammatory drugs on spinal fusion". Spine 36.7 (2011) E461-E468.

32. Kurd MF., et al. "The Role of Multimodal Analgesia in Spine Surgery". The Journal of the American Academy of Orthopaedic Surgeons 25.4 (2017): 260-268. 
33. Kim SI., et al. "Preemptive multimodal analgesia for postoperative pain management after lumbar fusion surgery: a randomized controlled trial”. European Spine Journal 25.5 (2016): 1614-1619.

34. Harle P., et al. "Elective surgery in rheumatic disease and immunosuppression: to pause or not". Rheumatology-London Then Oxford- British Society for Rheumatology 49.10 (2010): 1799-1800.

35. Bissar L., et al. "Perioperative management of patients with rheumatic diseases". The Open Rheumatology Journal 7 (2013): 42-50.

36. Krause ML and Matteson EL. "Perioperative management of the patient with rheumatoid arthritis". World Journal of Orthopedics 5.3 (2014): 283-291.

37. Cutolo M., et al. "Anti-inflammatory mechanisms of methotrexate in rheumatoid arthritis". Annals of the Rheumatic Diseases 60.8 (2001): 729-735.

38. Grennan DM., et al. "Methotrexate and early postoperative complications in patients with rheumatoid arthritis undergoing elective orthopaedic surgery". Annals of the Rheumatic Diseases 60.3 (2001): 214-217.

39. Murata K., et al. "Lack of increase in postoperative complications with low-dose methotrexate therapy in patients with rheumatoid arthritis undergoing elective orthopedic surgery". Modern Rheumatology 16.1 (2006): 14-19.

40. Lee MA., et al. "The Perioperative Use of Disease-Modifying and Biologic Therapies in Patients With Rheumatoid Arthritis Undergoing Elective Orthopedic Surgery". Orthopedics 33.4 (2010): 257-260.

41. Chao NJ. "Overview of Immunosuppressive Agents Used for Prevention of Graft-Versus-Host Disease”. Up To Date, edition. T.W. Post. Waltham, MA: UpToDate (2017).

42. Kumar A., et al. "Inflammatory bowel disease: perioperative pharmacological considerations". Mayo Clinic Proceedings 86.8 (2011): 748-757.

43. Fox RI. "Mechanism of action of hydroxychloroquine as an antirheumatic drug". Semin Arthritis Seminars in Arthritis and Rheumatism 23.2-1 (1993): 82-91.

44. Takeda K and Akira S. "Toll-like receptors". Current Protocols in Immunology 109 (2015): 1-10.

45. Bibbo C., et al. "The influence of rheumatoid chemotherapy, age, and presence of rheumatoid nodules on postoperative complications in rheumatoid foot and ankle surgery: analysis of 725 procedures in 104 patients [corrected]". Foot and Ankle International 24.1 (2003): 40-44.
46. Escalante A and Beardmore TD. "Risk factors for early wound complications after orthopedic surgery for rheumatoid arthritis". The Journal of Rheumatology 22.10 (1995): 1844-1851.

47. Goodman SM. "Rheumatoid arthritis: Perioperative management of biologics and DMARDs". Seminars in Arthritis and Rheumatism 44.6 (2015): 627-632.

48. Breedveld FC and Dayer JM. "Leflunomide: mode of action in the treatment of rheumatoid arthritis". Annals of the Rheumatic Diseases 59.11 (2000): 841-849.

49. Tanaka N., et al. "Examination of the risk of continuous leflunomide treatment on the incidence of infectious complications after joint arthroplasty in patients with rheumatoid arthritis". Journal of Clinical Rheumatology 9.2 (2003): 115-118.

50. Fuerst M., et al. "Leflunomide increases the risk of early healing complications in patients with rheumatoid arthritis undergoing elective orthopedic surgery". Rheumatology International 26.12 (2006): 1138-1142.

51. Volin MV., et al. "The effect of sulfasalazine on rheumatoid arthritic synovial tissue chemokine production". Experimental and Molecular Pathology 73.2 (2002): 84-92.

52. Den Broeder AA., et al. "Risk factors for surgical site infections and other complications in elective surgery in patients with rheumatoid arthritis with special attention for anti-tumor necrosis factor: a large retrospective study". The Journal of Rheumatology 34.4 (2007): 689-695.

53. Veetil BMA and Bongartz T. "Perioperative care for patients with rheumatic diseases". Nature Reviews Rheumatology 8.1 (2012): 32-41.

54. Vasanthi P., et al. "Role of tumor necrosis factor-alpha in rheumatoid arthritis: a review". International Journal of Rheumatic Diseases 10.4 (2007): 270-274.

55. Ma X and Xu S. "TNF inhibitor therapy for rheumatoid arthritis". Biomedical Reports 1.2 (2013): 177-184.

56. Dixon WG., et al. "Rates of serious infection, including sitespecific and bacterial intracellular infection, in rheumatoid arthritis patients receiving anti-tumor necrosis factor therapy: results from the British Society for Rheumatology Biologics Register". Arthritis and Rheumatology 54.8 (2006): 2368-2376.

57. Ruyssen-Witrand A., et al. "Complication rates of 127 surgical procedures performed in rheumatic patients receiving tumor necrosis factor alpha blockers". Clinical and Experimental Rheumatology 25.3 (2007): 430-436.

58. Schneeweiss S., et al. "Anti-tumor necrosis factor alpha therapy and the risk of serious bacterial infections in elderly patients with rheumatoid arthritis". Arthritis and Rheumatology 56.6 (2007): 1754-1764. 
59. Giles JT., et al. "Tumor necrosis factor inhibitor therapy and risk of serious postoperative orthopedic infection in rheumatoid arthritis". Arthritis and Rheumatology 55.2 (2006): 333337.

60. Cohen SB., et al. "Rituximab for rheumatoid arthritis refractory to anti-tumor necrosis factor therapy: results of a multicenter, randomized, double-blind, placebo-controlled, phase III trial evaluating primary efficacy and safety at twenty-four weeks". Arthritis and Rheumatology 54.9 (2006): 2793-2806.

61. Godot S., et al. "Safety of surgery after rituximab therapy in 133 patients with rheumatoid arthritis: data from the autoimmunity and rituximab registry". Arthritis Care and Research 65.11 (2013): 1874-1879.

62. Gardner GC. "Management of medications in patients with rheumatic diseases during the perioperative period". Perioperative Management of Patients with Rheumatic Disease (2012): 71-85.

\section{Assets from publication with us}

- Prompt Acknowledgement after receiving the article

- Thorough Double blinded peer review

- Rapid Publication

- Issue of Publication Certificate

- High visibility of your Published work

Website: https://www.actascientific.com/

Submit Article: https://www.actascientific.com/submission.php Email us: editor@actascientific.com

Contact us: +919182824667 